\title{
UHydropac/labloprodurets
}

\section{A new alternative watering system}

\author{
Frank J. Gianni, AA, BA, Med \& Marc A. Willis, BS, MBA, LATG
}

The Hydropac ${ }^{\mathrm{TM}}$ AWS-5000 Pouch Machine is an automatic form/fill/sealing machine designed to make and fill 8- and 13-oz. pouches using a continuous roll of special plastic film stock. One of the unique features of the Hydropac machine is that it can be connected to ordinary tap water, to an in-house water supply, or to a customized point-of-use treatment unit. A sterile disposable dripless single-use valve provides water to mice, rats, hamsters, and guinea pigs.

Laboratory animal management personnel must often balance the pressures of providing water to laboratory mice of the quality and quantity consistent with the institution's research mission and needs. A few of the variables that may determine which watering systems to use include cost containment, water quality, short- and long-term capital startup and expansion costs, and operational costs.

\section{Hydropac-the system}

The Hydropac system comprises three basic functional components: the Hydropac pouch production equipment (Fig. 1), material handling equipment, and the cage delivery equipment. All three components are necessary to deliver the Hydropac pouch system to laboratory rodents.

The Hydropac machine can produce an 8-oz. or 13-oz. pouch (Fig. 2a) at the operator's discretion. The 8-oz. pouches are suitable for facilities where water delivery vehicles and/or cages, by standard operating procedure, require changing every 7 days. In contrast, 13oz. pouches are suitable for those facilities where water delivery vehicles and/or cages are changed every 14 days. In both cases, the volume of water produced is more than sufficient for the typical consumption of as many as five adult laboratory mice per cage during those time frames, thereby requiring no intervention by the end user.

Not only does the Hydropac machine require little operator effort, but it is simple to learn and easy for one person to operate. Setup, system maintenance, and shutdown times vary with the quantity of pouches produced, and efficiency increases with the volume of pouches generated. This is primarily a result of the machine's capacity to generate large volumes of pouches in a relatively short time.

The machine operates on a fixed output rate, generating one pouch approximately every 2 seconds or 1,800 pouches per hour. For example, in a 5,000-cage facility, the total time to set up and run is 3.5 hours. A 50,000-cage facility would require 31.5 hours to complete the same task.

Optional equipment to handle the movement, storage, transportation (Fig. 2b), and disposal of the pouches is also available.

Hydropac/ Lab Products, Inc., 742 Sussex Ave., PO Box 639, Seaford, DE 19973. Correspondence should be addressed to F.J.G. (fgianni@comcast.net).

\section{Scalability and flexibility}

The decision to deliver a single global water treatment regimen to all laboratory rodents may be undesirable in specific research protocols. For example, if hyperchlorination is the Hydropac pouch water treatment of choice, the addition of chlorine may be undesirable in specific research studies, or specific research protocols may warrant the use of acidified water. The Hydropac system can provide for both. It is possible to easily modify source water treatments to deliver an array of solutions depending on each institution's individual needs.

If it is necessary to add chemicals such as experimental drugs to a small number of pouches, a stick-on silicone patch allows chemicals to be injected into the pouch using a syringe and needle.

\section{Water consumption statistics}

The volume of water provided to laboratory mice depends on many variables, including the method of water delivery, the water delivery

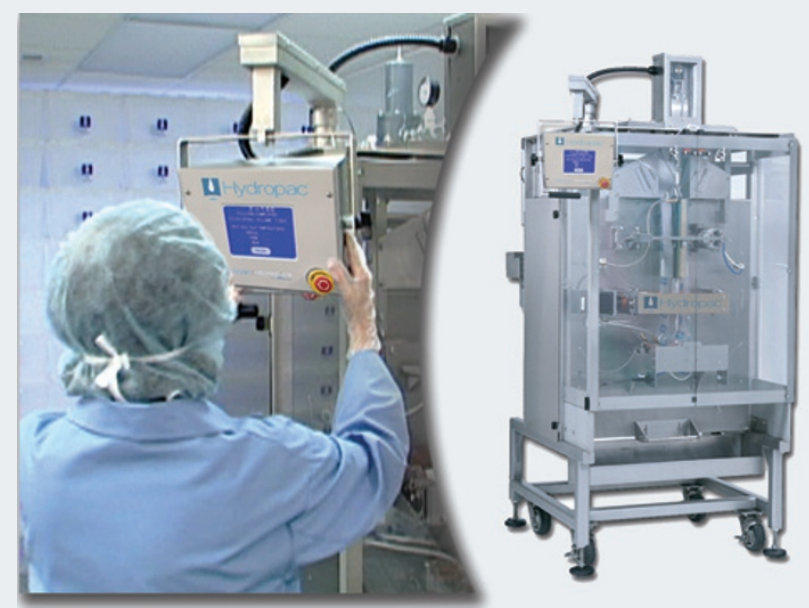

FIGURE 1 | The Hydropac AWS-5000 Pouch Machine automatically forms, fills, and seals pouches using a continuous roll of special plastic film stock. A touch screen panel located on an articulating arm activates all control functions. 

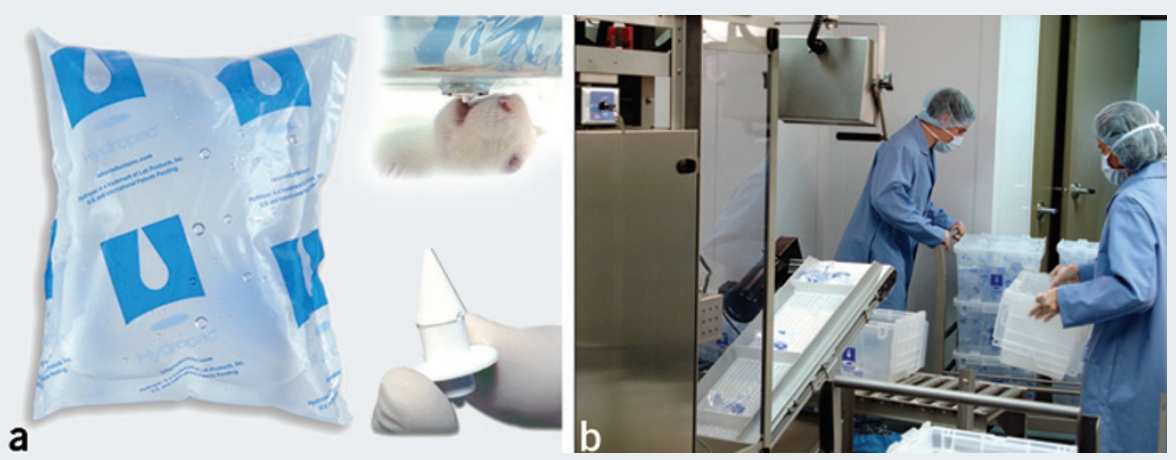

FIGURE 2 | The Hydropac AWS-5000 can produce 8-0z. and 13-0z. pouches. (a) A sterile disposable valve can provide water to mice, rats, hamsters, and guinea pigs. (b) Optional equipment, such as Tote Containers, is available for the movement, storage, and transportation of Hydropac pouches.

material. Other options include a pouch disposal compactor, and a cost-effective preventive maintenance and service contract. In terms of operational costs, Hydropac may offer the end user significant savings. The Hydropac machine requires little outsourced maintenance, because in-house staff can easily handle routine maintenance, potentially eliminating costly maintenance and service contracts. With Hydropac, bottle washing and filling equipment, bottle baskets, access to steam sterilizers, automated watering systems, and their associated components are not necessary to deliver water to laboratory mice.

\section{Hydropac reliability}

Failures can plague both water bottles

system used, and the water delivery vehicle used. Independent testing indicates both the 8-oz. and 13-oz. volumes are sufficient to meet the change-out frequencies of 7- and 14-day cycles, respectively.

\section{Water quality}

Many variables influence an institution's decision for water quality solutions. Municipal water supplies can be sources of undesirable microbial and chemical contaminants that define the water treatment regimen. However, source water quality may not be the only consideration. Changes in water quality (e.g., increases in microbal levels) can and may occur after water is delivered to the cage.

An independent study conducted by one research institution confirms the latter possibility. Serial water samples were harvested from pouches and conventional water delivery systems at days 1, 3, 7, and 14; control samples were harvested at days 0 and 14. Results indicated microbial growth in water bottle samples harvested at day 1 . The final free chlorine concentration in the pouch at the time of study initiation was 3 p.p.m. By day 14, water bottle culture results revealed microbial counts in excess of 1,000,000 microorganisms per ml. In stark contrast, all serial samples harvested from Hydropac pouches through day 14 showed no microbial growth.

\section{Hydropac - a cost reduction and containment solution}

Additional labor cost savings occur at the cage-changing level. The average pouch-changing time ranges from 10 to 15 seconds per cage and includes pouch removal, installation of a new pouch and valve, and pouch disposal. At a fully loaded labor rate of $\$ 18.00$ per hour, the labor cost to change and dispose averages $\$ 0.07$ per cage. This translates to an average labor cost of $\$ 0.005$ per day. In comparison, timeand-motion studies associated with water bottle processing, including bottle changing, washing, filling, sterilizing, and transfer, averaged 111.6 seconds per bottle. These data suggest that a 10-fold increase in labor exists over the equivalent time to handle Hydropac.

A single Hydropac machine can produce a sufficient volume of pouches to meet most operational needs. All that is required is the Hydropac machine, and disposable Hydropac valves and pouch and automated watering systems, resulting in cage flooding or water deprivation. At a minimum, the effort required to address water delivery system failures can be significant. In an independent study, Hydropac underwent comparative evaluation for system failures, which were defined as watering system events that require intervention by animal care staff ranging from excessively soiled cages due to a water system failure (leak), cage flooding, or water deprivation.

The cages assessed in one study included mice newly received from vendors, mice weaned from cages adapted to both Hydropac and water bottle/automated watering systems, and adult mice transferred from water bottles/automated watering systems to Hydropac. In this 1-month study period, there were 56 cage events using intracage automatic watering and 41 water bottle events, contrasted with only 6 Hydropac events.

These results suggest that Hydropac is more reliable than other water delivery systems. These data also suggest a labor savings benefit with Hydropac, because there are fewer events requiring animal caretaker and/or clinical intervention and fewer events during overtime or premium hours translate into reduced labor costs as well.

\section{Investigator and husbandry staff acceptance}

The movement of cages to and from the workbench for experimental manipulation can represent a real problem for both research and husbandry staff. The movement of cages with water bottles can lead to excessive cage soiling, thereby increasing the frequency of cage changing and, ultimately, labor costs. Likewise, separation of laboratory rodents on intracage automatic watering devices to new cages requires valve priming and particular attention to the delivery of water to the valve and its availability to cage occupants. Under these conditions, water remains in the Hydropac pouch when moved or handled and is immediately available to laboratory mice.

Copyright 2005. Hydropac and Disposable Valve are trademarks of Hydropac/Lab Products, Inc. Covered by various U.S. and International Patents and Patents pending. 